\title{
On the Generalized Cluster Algebras of Geometric Type
}

\author{
Liqian $B A I^{\dagger^{1}}$, Xueqing $C H E N{ }^{\dagger^{2}}$, Ming DING ${ }^{\dagger^{3}}$ and Fan $X U^{\dagger^{4}}$ \\ $\dagger^{1}$ School of Mathematics and Statistics, Northwestern Polytechnical University, \\ Xi'an, Shaanxi 710072, P. R. China \\ E-mail: bailiqian@nwpu.edu.cn \\ $\dagger^{2}$ Department of Mathematics, University of Wisconsin-Whitewater, \\ 800 West Main Street, Whitewater, WI 53190, USA \\ E-mail: chenx@uww.edu \\ $\dagger^{3}$ School of Mathematics and Information Science, Guangzhou University, \\ Guangzhou 510006, P. R. China \\ E-mail:m-ding04@mails.tsinghua.edu.cn \\ $\dagger^{4}$ Department of Mathematical Sciences, Tsinghua University, Beijing 100084, P. R. China \\ E-mail: fanxu@mail.tsinghua.edu.cn
}

Received April 01, 2020, in final form September 14, 2020; Published online September 28, 2020 https://doi.org/10.3842/SIGMA.2020.092

\begin{abstract}
We develop and prove the analogs of some results shown in [Berenstein A., Fomin S., Zelevinsky A., Duke Math. J. 126 (2005), 1-52] concerning lower and upper bounds of cluster algebras to the generalized cluster algebras of geometric type. We show that lower bounds coincide with upper bounds under the conditions of acyclicity and coprimality. Consequently, we obtain the standard monomial bases of these generalized cluster algebras. Moreover, in the appendix, we prove that an acyclic generalized cluster algebra is equal to the corresponding generalized upper cluster algebra without the assumption of the existence of coprimality.
\end{abstract}

Key words: cluster algebra; generalized cluster algebra; lower bound; upper bound; standard monomial

2020 Mathematics Subject Classification: 13F60; 05E16

Dedicated to our teacher Jie Xiao

on the occasion of his sixtieth birthday

\section{Background}

Fomin and Zelevinsky invented the concept of cluster algebras $[5,6]$ in order to create an algebraic framework for studying total positivity in algebraic groups and canonical bases in quantum groups. As a natural generalization, Chekhov and Shapiro introduced the generalized cluster algebras which arise from the Teichmüller spaces of Riemann surfaces with orbifold points [4]. The main difference between cluster algebras and generalized cluster algebras is that the binomial exchange relations for cluster variables of cluster algebras are replaced by the multinomial exchange relations for those cluster variables of generalized cluster algebras. In [4], Chekhov and Shapiro have shown that the generalized cluster algebras possess the remarkable Laurent phenomenon. Many other important properties of cluster algebras are also shown to hold in the generalized cluster algebras such as finite type classification, $g$-vectors and $F$-polynomials [4, 13$]$.

This paper is a contribution to the Special Issue on Cluster Algebras. The full collection is available at https://www.emis.de/journals/SIGMA/cluster-algebras.html 
Motivated by the Laurent phenomenon established in [5], Berenstein, Fomin and Zelevinsky in [2] introduced the notion of an upper cluster algebra, which is a certain (maybe infinitely many) intersection of Laurent polynomial rings. The upper bound is a certain finite intersection of rings of Laurent polynomials. The lower bound is the subalgebra of a cluster algebra generated by specific finitely many cluster variables. These four algebras satisfy the following relations:

lower bound $\subseteq$ cluster algebra $\subseteq$ upper cluster algebra $\subseteq$ upper bound.

They proved that for a cluster algebra possessing an acyclic and coprime seed, its lower bound coincides with its upper bound, thus, all four algebras coincide. The standard monomial bases of these kinds of cluster algebras can then be naturally constructed. Beyond acyclic cluster algebras, Muller [11] introduced the notion of locally acyclic cluster algebras, and it turns out that locally acyclic cluster algebras coincide with their upper cluster algebras [11, 12]. Bucher, Machacek and Shapiro [3] discussed how the choice of the ground ring impacts whether a cluster algebra is equal to its upper cluster algebra.

From a geometric point of view, upper cluster algebras are more natural than cluster algebras $[1,8]$. In [10], Matherne and Muller provided techniques for producing explicit presentations of upper cluster algebras. Plamondon [14] used quiver representations to obtain a formula for certain elements of skew-symmetric upper cluster algebras. Lee, Li and Mills [9] developed an elementary formula for certain non-trivial elements of an upper cluster algebra with positive coefficients, and proved that these elements form a basis of an acyclic cluster algebra.

Gekhtman, Shapiro and Vainshtein [7] proved that generalized upper cluster algebras over certain rings retain all properties of ordinary upper cluster algebras, and under certain coprimality conditions coincide with the intersection of rings of Laurent polynomials in a finite collection of clusters.

The aim of this paper is to continue the investigation of the structure of generalized cluster algebras. By using the methods developed in [2], we prove that the conditions of acyclicity and coprimality close the gap between lower bounds and upper bounds associated to generalized cluster algebras as the extension of the similar results of ordinary cluster algebras. Consequently, we obtain the standard monomial bases of these generalized cluster algebras. It would be desirable to apply the results that we obtain in this paper to construct some good bases of the corresponding generalized cluster algebras in the future work.

In the appendix, we extend Muller's results on acyclic and locally acyclic cluster algebras to acyclic generalized cluster algebras. By using the same arguments as given in [12], we prove that acyclic generalized cluster algebras coincide with their generalized upper cluster algebras without the assumption of the existence of coprimality.

\section{Preliminaries}

First, let us recall the definition of the generalized cluster algebras of geometric type (see $[4,7])$.

In the following, we use $[i, j]$ to denote the set $\{i, i+1, \ldots, j-1, j\}$ for integers $i<j$. Let $m$ and $n$ be positive integers with $m \geq n$. An $n \times n$ matrix $B$ is called skew-symmetrizable if there exists a diagonal matrix $D=\operatorname{diag}\left(\widetilde{d}_{1}, \widetilde{d}_{2}, \ldots, \widetilde{d}_{n}\right)$, where $\widetilde{d}_{i}$ are positive integers for all $i \in[1, n]$, such that $D B$ is skew-symmetric. Let ex be a subset of $[1, m]$ with $|\mathbf{e x}|=n$. Let $\widetilde{B}=\left(b_{i j}\right)$ be an $m \times n$ integer matrix such that $\widetilde{B}$ has the $n \times n$ skew-symmetrizable submatrix $B$ with rows labeled by ex. The columns of $\widetilde{B}$ are also labeled by ex. The matrix $B$ is called the principal part of $\widetilde{B}$. For each $s \in \mathbf{e x}$, let $d_{s}$ be a positive integer such that $d_{s}$ divides $b_{i s}$ for any $i \in[1, m]$, and let $\beta_{i s}:=\frac{b_{i s}}{d_{s}}$.

Let us denote by $\mathbb{Q}\left(x_{1}, x_{2}, \ldots, x_{m}\right)$ the function field of $m$ variables over $\mathbb{Q}$ with a transcendence basis $\left\{x_{1}, x_{2}, \ldots, x_{m}\right\}$. Let the coefficient group $\mathbb{P}$ be the multiplicative free abelian group 
generated by $\left\{x_{i} \mid i \in[1, m]-\mathbf{e x}\right\}$ and $\mathbb{Z P}$ be its integer group ring. For each $i \in \mathbf{e x}$, we will denote by $\rho_{i}:=\left\{\rho_{i, 0}, \ldots, \rho_{i, d_{i}}\right\}$ the $i$ th string, where $\rho_{i, 0}=\rho_{i, d_{i}}=1$ and $\rho_{i, j}$ are monomials in $\mathbb{Z}\left[x_{i} \mid i \in[1, m]-\mathbf{e x}\right]$ for $1 \leq j \leq d_{i}-1$.

Definition 2.1. A generalized seed (of geometric type) is a triple $(\widetilde{\mathbf{x}}, \rho, \widetilde{B})$, where

(1) the set $\widetilde{\mathbf{x}}=\left\{x_{1}, x_{2}, \ldots, x_{m}\right\}$ is called the extended cluster and the set $\mathbf{x}=\left\{x_{i} \mid i \in \mathbf{e x}\right\}$ is called the cluster whose elements are called cluster variables, and the elements of $\left\{x_{i} \mid i \in\right.$ $[1, m]-\mathbf{e x}\}$ are called frozen variables;

(2) $\rho=\left\{\rho_{i} \mid i \in \mathbf{e x}\right\}$ is the set of strings;

(3) the matrices $\widetilde{B}$ and $B$ are called the extended exchange matrix and the exchange matrix, respectively.

Define the function $[x]_{+}:=x$ if $x \geq 0$, and $[x]_{+}:=0$ otherwise.

Definition 2.2. For $i \in \underset{\widetilde{e x}}{\mathbf{e x}}$, the mutation of a generalized seed $(\widetilde{\mathbf{x}}, \rho, \widetilde{B})$ in direction $i$ is another generalized seed $\mu_{i}(\widetilde{\mathbf{x}}, \rho, \widetilde{B}):=\left(\widetilde{\mathbf{x}}^{\prime}, \rho^{\prime}, \widetilde{B}^{\prime}\right)$, where

(1) the set $\widetilde{\mathbf{x}}^{\prime}:=\left(\widetilde{\mathbf{x}}-\left\{x_{i}\right\}\right) \cup\left\{x_{i}^{\prime}\right\}$ with

$$
x_{i}^{\prime}:=\mu_{i}\left(x_{i}\right)=x_{i}^{-1}\left(\sum_{r=0}^{d_{i}} \rho_{i, r} \prod_{j=1}^{m} x_{j}^{r\left[\beta_{j i}\right]_{+}+\left(d_{i}-r\right)\left[-\beta_{j i}\right]_{+}}\right),
$$

which is called the exchange relation;

(2) $\rho^{\prime}:=\mu_{i}(\rho)=\left\{\rho_{i}^{\prime}, \rho_{j} \mid j \in \mathbf{e x}-\{i\}\right\}$, where $\rho_{i}^{\prime}=\left\{\rho_{i, 0}^{\prime}, \ldots, \rho_{i, d_{i}}^{\prime}\right\}$ such that $\rho_{i, r}^{\prime}=\rho_{i, d_{i}-r}$ for $0 \leq r \leq d_{i}$;

(3) the matrix $\widetilde{B}^{\prime}:=\mu_{i}(\widetilde{B})$ is defined by

$$
b_{k l}^{\prime}= \begin{cases}-b_{k l} & \text { if } k=i \quad \text { or } \quad l=i \\ b_{k l}+\left(\left[b_{i l}\right]_{+} b_{k i}+b_{i l}\left[-b_{k i}\right]_{+}\right) & \text {otherwise }\end{cases}
$$

Note that $\mu_{i}$ is an involution. The generalized seed $(\widetilde{\mathbf{y}}, \varepsilon, \widetilde{A})$ is said to be mutation-equivalent to $(\widetilde{\mathbf{x}}, \rho, \widetilde{B})$, if $(\widetilde{\mathbf{y}}, \varepsilon, \widetilde{A})$ can be obtained from $(\widetilde{\mathbf{x}}, \rho, \widetilde{B})$ by a sequence of seed mutations. If $d_{r}=1$ for all $r \in \mathbf{e x}$, then one recovers the ordinary cluster algebras.

Example 2.3 ([4, Theorem 2.7]). Let $\widetilde{\mathbf{x}}=\mathbf{x}=\left\{x_{1}, x_{2}\right\}$ and $\widetilde{B}=B=\left(\begin{array}{rr}0 & 1 \\ -2 & 0\end{array}\right)$. Let $\left(d_{1}, d_{2}\right)=$ $(2,1)$ and $\rho=\left\{\rho_{1}, \rho_{2}\right\}$, where $\rho_{1}=\{1, h, 1\}$ for any $h \in \mathbb{Z}$ and $\rho_{2}=\{1,1\}$. The triple $(\mathbf{x}, \rho, B)$ is a generalized seed. Let $B^{\prime}=\left(\begin{array}{cc}0 & -1 \\ 2 & 0\end{array}\right)$. Then we have

$$
\begin{aligned}
\cdots & \stackrel{\mu_{1}}{\longleftrightarrow}\left(\left\{x_{1}, x_{0}\right\}, \rho, B^{\prime}\right) \stackrel{\mu_{2}}{\longleftrightarrow}\left(\left\{x_{1}, x_{2}\right\}, \rho, B\right) \stackrel{\mu_{1}}{\longleftrightarrow}\left(\left\{x_{3}, x_{2}\right\}, \rho, B^{\prime}\right) \\
& \stackrel{\mu_{2}}{\longleftrightarrow}\left(\left\{x_{3}, x_{4}\right\}, \rho, B\right) \stackrel{\mu_{1}}{\longleftrightarrow}\left(\left\{x_{5}, x_{4}\right\}, \rho, B^{\prime}\right) \stackrel{\mu_{2}}{\longleftrightarrow}\left(\left\{x_{5}, x_{6}\right\}, \rho, B\right) \\
& \stackrel{\mu_{1}}{\longleftrightarrow}\left(\left\{x_{7}, x_{6}\right\}, \rho, B^{\prime}\right) \stackrel{\mu_{2}}{\longleftrightarrow}\left(\left\{x_{7}, x_{8}\right\}, \rho, B\right) \stackrel{\mu_{1}}{\longleftrightarrow} \cdots,
\end{aligned}
$$

where the cluster variables $x_{k}$ for $k \in \mathbb{Z}$ satisfy the exchange relations:

$$
x_{k-1} x_{k+1}= \begin{cases}1+x_{k} & \text { if } k \text { is odd } \\ 1+h x_{k}+x_{k}^{2} & \text { if } k \text { is even }\end{cases}
$$

By a direct calculation, we have that

$$
x_{3}=x_{1}^{-1}+h x_{1}^{-1} x_{2}+x_{1}^{-1} x_{2}^{2},
$$




$$
\begin{aligned}
& x_{4}=x_{2}^{-1}+x_{1}^{-1} x_{2}^{-1}+h x_{1}^{-1}+x_{1}^{-1} x_{2}, \\
& x_{5}=x_{1}^{-1}+h x_{1}^{-1} x_{2}^{-1}+h x_{2}^{-1}+x_{1}^{-1} x_{2}^{-2}+2 x_{2}^{-2}+x_{1} x_{2}^{-2}, \\
& x_{6}=x_{0}=x_{2}^{-1}+x_{1} x_{2}^{-1}, \quad x_{7}=x_{1}, \quad x_{8}=x_{2} .
\end{aligned}
$$

It is now clear that the sequence of cluster variables $\left\{x_{k}\right\}_{k \in \mathbb{Z}}$ is 6 -periodic. Thus we have only six distinct cluster variables.

Definition 2.4. For an initial generalized seed $(\widetilde{\mathbf{x}}, \rho, \widetilde{B})$, the generalized cluster algebra $\mathcal{A}(\widetilde{\mathbf{x}}, \rho, \widetilde{B})$ is the $\mathbb{Z} \mathbb{P}$-subalgebra of $\mathbb{Q}\left(x_{1}, \ldots, x_{m}\right)$ generated by all cluster variables from all generalized seeds which are mutation-equivalent to $(\widetilde{\mathbf{x}}, \rho, \widetilde{B})$. The integer $n$ is the $\operatorname{rank}$ of $\mathcal{A}(\widetilde{\mathbf{x}}, \rho, \widetilde{B})$.

For each $i \in \mathbf{e x}$, we define

$$
P_{i}:=x_{i} x_{i}^{\prime}=\sum_{r=0}^{d_{i}} \rho_{i, r} \prod_{j=1}^{m} x_{j}^{r\left[\beta_{j i}\right]_{+}+\left(d_{i}-r\right)\left[-\beta_{j i}\right]_{+}} .
$$

It follows that $P_{i} \in \mathbb{Z} \mathbb{P}\left[x_{1}, \ldots, x_{i-1}, x_{i+1}, \ldots, x_{n}\right]$.

Definition 2.5. The generalized seed $(\widetilde{\mathbf{x}}, \rho, \widetilde{B})$ is called coprime if $P_{i}$ and $P_{j}$ are coprime for any two different $i, j \in \mathbf{e x}$.

Let $(\widetilde{\mathbf{x}}, \rho, \widetilde{B})$ be a generalized seed. The directed graph $\Gamma(\widetilde{\mathbf{x}}, \rho, \widetilde{B})$ is defined as follows:

(1) its vertices consist of all $i \in \mathbf{e x ;}$

(2) a pair $(i, j)$ is a directed edge of $\Gamma(\widetilde{\mathbf{x}}, \rho, \widetilde{B})$ if and only if $b_{i j}>0$.

Definition 2.6. The generalized seed $(\widetilde{\mathbf{x}}, \rho, \widetilde{B})$ is called acyclic if $\Gamma(\widetilde{\mathbf{x}}, \rho, \widetilde{B})$ does not contain any oriented cycle. A generalized cluster algebra $\mathcal{A}$ is called acyclic if it has an acyclic generalized seed.

The following definition is a natural generalization of [2, Definition 1.15].

Definition 2.7. Let $(\widetilde{\mathbf{x}}, \rho, \widetilde{B})$ be a generalized seed. A standard monomial in $\left\{x_{i}, x_{i}^{\prime} \mid i \in \mathbf{e x}\right\}$ is a monomial that does not have any factor of the form $x_{i} x_{i}^{\prime}$ for any $i \in \mathbf{e x}$.

In order to define the upper bounds and lower bounds, we write $\mathbf{e x}=\left\{i_{1}, \ldots, i_{n}\right\}$.

Definition 2.8. For a generalized seed $(\widetilde{\mathbf{x}}, \rho, \widetilde{B})$, the upper bound is defined by

$$
\mathcal{U}(\widetilde{\mathbf{x}}, \rho, \widetilde{B}):=\mathbb{Z} \mathbb{P}\left[x_{i_{1}}^{ \pm 1}, \ldots, x_{i_{n}}^{ \pm 1}\right] \cap \bigcap_{k=1}^{n} \mathbb{Z} \mathbb{P}\left[x_{i_{1}}^{ \pm 1}, \ldots, x_{i_{k-1}}^{ \pm 1},\left(x_{i_{k}}^{\prime}\right)^{ \pm 1}, x_{i_{k+1}}^{ \pm 1}, \ldots, x_{i_{n}}^{ \pm 1}\right]
$$

and the lower bound by

$$
\mathcal{L}(\widetilde{\mathbf{x}}, \rho, \widetilde{B}):=\mathbb{Z} \mathbb{P}\left[x_{i_{1}}, x_{i_{1}}^{\prime}, \ldots, x_{i_{n}}, x_{i_{n}}^{\prime}\right] .
$$

Note that

$$
\mathcal{L}(\widetilde{\mathbf{x}}, \rho, \widetilde{B}) \subseteq \mathcal{A}(\widetilde{\mathbf{x}}, \rho, \widetilde{B}) \subseteq \mathcal{U}(\widetilde{\mathbf{x}}, \rho, \widetilde{B}) .
$$

Theorem 2.9 ([7, Theorem 4.1]). Let $i \in \mathbf{e x . ~ I f ~ t h e ~ g e n e r a l i z e d ~ s e e d s ~}(\widetilde{\mathbf{x}}, \rho, \widetilde{B})$ and $\mu_{i}(\widetilde{\mathbf{x}}, \rho, \widetilde{B})$ are coprime, then we have

$$
\mathcal{U}(\widetilde{\mathbf{x}}, \rho, \widetilde{B})=\mathcal{U}\left(\mu_{i}(\widetilde{\mathbf{x}}, \rho, \widetilde{B})\right) .
$$


The following definition is a generalization of [12, Section 3.1].

Definition 2.10. Let $(\widetilde{\mathbf{x}}, \rho, \widetilde{B})$ be a generalized seed and $i \in$ ex. A new generalized seed $\left(\widetilde{\mathbf{x}}^{\dagger}, \rho^{\dagger}, \widetilde{B}^{\dagger}\right)$ is defined as follows:

(1) let $\mathbb{Z} \mathbb{P}^{\dagger}=\mathbb{Z} \mathbb{P}\left[x_{i}^{ \pm 1}\right], \mathbf{x}^{\dagger}=\left\{x_{k} \mid k \in \mathbf{e x}-\{i\}\right\}$ and $\widetilde{\mathbf{x}}^{\dagger}-\mathbf{x}^{\dagger}=(\widetilde{\mathbf{x}}-\mathbf{x}) \cup\left\{x_{i}\right\}$;

(2) the matrix $\widetilde{B}^{\dagger}$ is obtained from $\widetilde{B}$ by deleting the $i$ th column, the principal part $B^{\dagger}$ is the submatrix of $\widetilde{B}^{\dagger}$ with rows labeled by ex $-\{i\}$;

(3) let $\rho^{\dagger}=\rho-\left\{\rho_{i}\right\}$.

The generalized seed $\left(\widetilde{\mathbf{x}}^{\dagger}, \rho^{\dagger}, \widetilde{B}^{\dagger}\right)$ is called the freezing of $(\widetilde{\mathbf{x}}, \rho, \widetilde{B})$ at $x_{i}$. The freezing of $\mathcal{A}(\widetilde{\mathbf{x}}, \rho, \widetilde{B})$ at $x_{i}$ is defined to be the generalized cluster algebra $\mathcal{A}\left(\widetilde{\mathbf{x}}^{\dagger}, \rho^{\dagger}, \widetilde{B}^{\dagger}\right)$, which is the $\mathbb{Z} \mathbb{P}^{\dagger}$-subalgebra of $\mathbb{Q}\left(x_{1}, \ldots, x_{m}\right)$ generated by all cluster variables from the generalized seeds which are mutationequivalent to $\left(\widetilde{\mathbf{x}}^{\dagger}, \rho^{\dagger}, \widetilde{B}^{\dagger}\right)$.

Example 2.11. Let $(\mathbf{x}, \rho, B)$ be the generalized seed from Example 2.3. Let $\mathbf{x}^{\dagger}=\left\{x_{2}\right\}, \rho^{\dagger}=$ $\left\{\rho_{2}\right\}$ and $B^{\dagger}=(0)$. Then the generalized seed $\left(\mathbf{x}^{\dagger}, \rho^{\dagger}, B^{\dagger}\right)$ is the freezing of $(\mathbf{x}, \rho, B)$ at $x_{1}$. It follows that the generalized cluster algebra $\mathcal{A}\left(\mathbf{x}^{\dagger}, \rho^{\dagger}, B^{\dagger}\right)=\mathbb{Z}\left[x_{1}^{ \pm 1}, x_{2}, \frac{x_{1}+1}{x_{2}}\right]$ is the freezing of $\mathcal{A}(\mathbf{x}, \rho, B)$ at $x_{1}$. Similarly, the generalized seed $\left(\left\{x_{1}\right\},\left\{\rho_{1}\right\},(0)\right)$ is the freezing of $(\mathbf{x}, \rho, B)$ at $x_{2}$ and the freezing of $\mathcal{A}(\mathbf{x}, \rho, B)$ at $x_{2}$ is $\mathcal{A}\left(\left\{x_{1}\right\},\left\{\rho_{1}\right\},(0)\right)=\mathbb{Z}\left[x_{1}, x_{2}^{ \pm 1}, \frac{1+h x_{2}+x_{2}^{2}}{x_{1}}\right]$.

The freezing at $x_{i}$ is compatible with the mutation in direction $j$ for $i \neq j$, therefore we have the following result.

Lemma 2.12. Let $i, j \in$ ex be distinct and assume $\mu_{j}(\widetilde{\mathbf{x}}, \rho, \widetilde{B})=(\widetilde{\mathbf{y}}, \varepsilon, \widetilde{A})$. If $\left(\widetilde{\mathbf{x}}^{\dagger}, \rho^{\dagger}, \widetilde{B}^{\dagger}\right)$ is the freezing of $(\widetilde{\mathbf{x}}, \rho, \widetilde{B})$ at $x_{i}$ and $\left(\widetilde{\mathbf{y}}^{\dagger}, \varepsilon^{\dagger}, \widetilde{A}^{\dagger}\right)$ the freezing of $(\widetilde{\mathbf{y}}, \varepsilon, \widetilde{A})$ at $y_{i}$, then we have that $\mu_{j}\left(\widetilde{\mathbf{x}}^{\dagger}, \rho^{\dagger}, \widetilde{B}^{\dagger}\right)=\left(\widetilde{\mathbf{y}}^{\dagger}, \varepsilon^{\dagger}, \widetilde{A}^{\dagger}\right)$.

Proof. The proof is straightforward, so we omit the details.

The freezing of $(\widetilde{\mathbf{x}}, \rho, \widetilde{B})$ at $\left\{x_{j_{1}}, \ldots, x_{j_{k}}\right\} \subsetneq \mathbf{x}$ is the generalized seed obtained from $(\widetilde{\mathbf{x}}, \rho, \widetilde{B})$ by iterated freezing at each cluster variable in $\left\{x_{j_{1}}, \ldots, x_{j_{k}}\right\}$ in any order. Note that the freezing at $\left\{x_{j_{1}}, \ldots, x_{j_{k}}\right\}$ is compatible with the mutation in direction $l$ for $l \notin\left\{j_{1}, \ldots, j_{k}\right\}$. It will cause no confusion if we still use $\left(\widetilde{\mathbf{x}}^{\dagger}, \rho^{\dagger}, \widetilde{B}^{\dagger}\right)$ to denote the resulting generalized seed. In the same manner, the generalized cluster algebra $\mathcal{A}\left(\widetilde{\mathbf{x}}^{\dagger}, \rho^{\dagger}, \widetilde{B}^{\dagger}\right)$ is called the freezing of $\mathcal{A}(\widetilde{\mathbf{x}}, \rho, \widetilde{B})$ at $\left\{x_{j_{1}}, \ldots, x_{j_{k}}\right\}$. The rank of $\mathcal{A}\left(\widetilde{\mathbf{x}}^{\dagger}, \rho^{\dagger}, \widetilde{B}^{\dagger}\right)$ is $n-k$.

\section{Lower bounds and upper bounds}

We follow the arguments in [2] to prove that lower bounds and upper bounds coincide under the assumptions of acyclicity and coprimality. We once defined the initial ex to be any subset of $[1, m]$ with $|\mathbf{e x}|=n$ in order to easily describe the freezing in Definition 2.10. For the sake of convenience, for the remainder of the paper, we will take $\mathbf{e x}=[1, n]$ after renumbering the indices of the original ex. Let $(\widetilde{\mathbf{x}}, \rho, \widetilde{B})$ be a generalized seed and $B=\left(b_{i j}\right)$ the corresponding principal part. Recall that $(\widetilde{\mathbf{x}}, \rho, \widetilde{B})$ is acyclic if and only if there exists a permutation $\sigma \in S_{n}$ such that $b_{\sigma(l), \sigma(k)} \geq 0$ for $1 \leqq k<l \leq n$. Hence we can assume that $b_{l k} \geq 0$ for $1 \leq k<l \leq n$ if the generalized seed $(\widetilde{\mathbf{x}}, \rho, \widetilde{B})$ is acyclic.

Theorem 3.1. If the generalized seed $(\widetilde{\mathbf{x}}, \rho, \widetilde{B})$ is acyclic, then the standard monomials in $x_{1}$, $x_{1}^{\prime}, \ldots, x_{n}, x_{n}^{\prime}$ are $\mathbb{Z} \mathbb{P}$-linearly independent in $\mathcal{L}(\widetilde{\mathbf{x}}, \rho, \widetilde{B})$. 
Proof. By using the same technique as in [2], we can prove the statement and we write the proof down here for readers' convenience.

For any $\mathbf{a}=\left(a_{1}, \ldots, a_{n}\right) \in \mathbb{Z}^{n}$, we denote by

$$
\mathbf{x}^{(\mathbf{a})}=x_{1}^{\left(a_{1}\right)} x_{2}^{\left(a_{2}\right)} \cdots x_{n}^{\left(a_{n}\right)}
$$

the standard monomial in $\left\{x_{1}, x_{1}^{\prime}, \ldots, x_{n}, x_{n}^{\prime}\right\}$, where $x_{i}^{\left(a_{i}\right)}=x_{i}^{a_{i}}$ if $a_{i} \geq 0$, and $x_{i}^{\left(a_{i}\right)}=\left(x_{i}^{\prime}\right)^{-a_{i}}$ if $a_{i}<0$, and we denote the Laurent monomial $x_{1}^{a_{1}} x_{2}^{a_{2}} \cdots x_{n}^{a_{n}}$ by $\mathbf{x}^{\mathbf{a}}$.

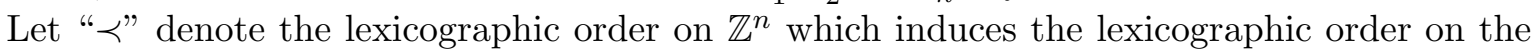
Laurent monomials as

$$
\mathbf{x}^{\mathbf{a}} \prec \mathbf{x}^{\mathbf{a}^{\prime}} \quad \text { if } \quad \mathbf{a} \prec \mathbf{a}^{\prime} .
$$

Note that $x_{i}^{(-1)}=x_{i}^{\prime}=x_{i}^{-1} P_{i}$ for $i \in[1, n]$. Using the assumption that $b_{l k} \geq 0$ for $l>k$, it follows that the lexicographically first monomial which appears in $x_{i}^{(-1)}$ is $x_{i}^{-1} \prod_{j=i+1}^{n} x_{j}^{b_{j i}} \prod_{k=n+1}^{m} x_{k}^{\left[b_{k i}\right]_{+}}$.

We then conclude that the lexicographically first monomial that appears in $\mathbf{x}^{\left(\mathbf{a}^{\prime}\right)}$ is preceded by the one in $\mathbf{x}^{(\mathbf{a})}$ if $\mathbf{a} \prec \mathbf{a}^{\prime}$. Therefore the standard monomials in $\left\{x_{1}, x_{1}^{\prime}, \ldots, x_{n}, x_{n}^{\prime}\right\}$ are $\mathbb{Z} \mathbb{P}$-linearly independent.

Remark 3.2. It is to be expected that the converse of the above theorem, i.e., "For a generalized seed $(\widetilde{\mathbf{x}}, \rho, \widetilde{B})$, if the standard monomials in $x_{1}, x_{1}^{\prime}, \ldots, x_{n}, x_{n}^{\prime}$ are $\mathbb{Z} \mathbb{P}$-linearly independent in $\mathcal{L}(\widetilde{\mathbf{x}}, \rho, \widetilde{B})$, then the generalized seed $(\widetilde{\mathbf{x}}, \rho, \widetilde{B})$ is acyclic" is also true as it was proved in $[2$, Proposition 5.1] for cluster algebras. But we have not been able to prove this.

The following two lemmas are generalizations of [2, Lemmas 4.1 and 4.2]. The proofs are omitted as they are similar to proofs in [2].

Lemma 3.3. We have that

$$
\mathcal{U}(\widetilde{\mathbf{x}}, \rho, \widetilde{B})=\bigcap_{i=1}^{n} \mathbb{Z} \mathbb{P}\left[x_{1}^{ \pm 1}, \ldots, x_{i-1}^{ \pm 1}, x_{i}, x_{i}^{\prime}, x_{i+1}^{ \pm 1}, \ldots, x_{n}^{ \pm 1}\right] .
$$

Lemma 3.4. Given $y \in \mathbb{Q}\left(x_{1}, \ldots, x_{m}\right)$, the element $y \in \mathbb{Z} \mathbb{P}\left[x_{1}, x_{1}^{\prime}, x_{2}^{ \pm 1}, \ldots, x_{n}^{ \pm 1}\right]$ if and only if $y=\sum_{k=a}^{b} c_{k} x_{1}^{k}(a \leq b)$ satisfies one of the following conditions:

(1) if $a \geq 0$, we have $c_{k} \in \mathbb{Z} \mathbb{P}\left[x_{2}^{ \pm 1}, \ldots, x_{n}^{ \pm 1}\right]$;

(2) if $a<0$, we have that $c_{k} \in \mathbb{Z} \mathbb{P}\left[x_{2}^{ \pm 1}, \ldots, x_{n}^{ \pm 1}\right]$ and $c_{k} P_{1}^{k} \in \mathbb{Z} \mathbb{P}\left[x_{2}^{ \pm 1}, \ldots, x_{n}^{ \pm 1}\right]$ for $k<0$ (namely, $c_{k}$ is divisible by $P_{1}^{|k|}$ in $\mathbb{Z} \mathbb{P}\left[x_{2}^{ \pm 1}, \ldots, x_{n}^{ \pm 1}\right]$ ).

Let $\mathbb{Z} \mathbb{P}^{\text {st }}\left[x_{2}, x_{2}^{\prime}, \ldots, x_{n}, x_{n}^{\prime}\right] \subseteq \mathbb{Z} \mathbb{P}\left[x_{2}, x_{2}^{\prime}, \ldots, x_{n}, x_{n}^{\prime}\right]$ denote the $\mathbb{Z} \mathbb{P}$-linear space spanned by the standard monomials in $\left\{x_{2}, x_{2}^{\prime}, \ldots, x_{n}, x_{n}^{\prime}\right\}$. Similarly, we denote by

$$
\mathbb{Z P}^{\mathrm{st}}\left[x_{1}, x_{2}, x_{2}^{\prime}, \ldots, x_{n}, x_{n}^{\prime}\right]
$$

the $\mathbb{Z} \mathbb{P}\left[x_{1}\right]$-linear space spanned by the standard monomials in $\left\{x_{2}, x_{2}^{\prime}, \ldots, x_{n}, x_{n}^{\prime}\right\}$. Using the exchange relations

$$
x_{i} x_{i}^{\prime}=P_{i} \in \mathbb{Z} \mathbb{P}\left[x_{1}, \ldots, x_{i-1}, x_{i+1}, \ldots, x_{n}\right]
$$


repeatedly, it follows that any element in $\mathbb{Z} \mathbb{P}\left[x_{2}, x_{2}^{\prime}, \ldots, x_{n}, x_{n}^{\prime}\right]$ is a $\mathbb{Z} \mathbb{P}\left[x_{1}\right]$-linear combination of standard monomials in $x_{2}, x_{2}^{\prime}, \ldots, x_{n}, x_{n}^{\prime}$. Thus, we have

$$
\mathbb{Z} \mathbb{P}\left[x_{2}, x_{2}^{\prime}, \ldots, x_{n}, x_{n}^{\prime}\right] \subseteq \mathbb{Z} \mathbb{P}^{\mathrm{st}}\left[x_{1}, x_{2}, x_{2}^{\prime}, \ldots, x_{n}, x_{n}^{\prime}\right] .
$$

Note that, this relation is the analogue of [2, equation (6.1)].

Define

$$
f_{1}: \mathbb{Z} \mathbb{P}\left[x_{2}, x_{2}^{\prime}, \ldots, x_{n}, x_{n}^{\prime}\right] \rightarrow \mathbb{Z} \mathbb{P}\left[x_{1}, x_{2}^{ \pm 1}, \ldots, x_{n}^{ \pm 1}\right] \quad \text { by } \quad x_{i} \mapsto x_{i} \quad \text { and } \quad x_{i}^{\prime} \mapsto x_{i}^{-1} P_{i}
$$

and

$$
f_{2}: \quad \mathbb{Z} \mathbb{P}\left[x_{1}, x_{2}^{ \pm 1}, \ldots, x_{n}^{ \pm 1}\right] \rightarrow \mathbb{Z} \mathbb{P}\left[x_{2}^{ \pm 1}, \ldots, x_{n}^{ \pm 1}\right] \quad \text { by } \quad x_{1} \mapsto 0 \quad \text { and } \quad x_{i}^{ \pm 1} \mapsto x_{i}^{ \pm 1} .
$$

Both are algebra homomorphisms, and consequently,

$$
f:=f_{2} \circ f_{1}: \mathbb{Z} \mathbb{P}\left[x_{2}, x_{2}^{\prime}, \ldots, x_{n}, x_{n}^{\prime}\right] \rightarrow \mathbb{Z P}\left[x_{2}^{ \pm 1}, \ldots, x_{n}^{ \pm 1}\right]
$$

is also an algebra homomorphism.

Given $y \in \mathbb{Z} \mathbb{P}\left[x_{1}^{ \pm 1}, \ldots, x_{n}^{ \pm 1}\right]$, as in [2, Definition 6.3], we also define the leading term LT $(y)$ of $y$ with respect to $x_{1}$ to be the sum of Laurent monomials with the smallest power of $x_{1}$, which are obtained from the Laurent expression of $y$ with non-zero coefficient.

The following results parallel to [2, Lemmas 6.2,6.4 and 6.5] can be obtained similarly.

Lemma 3.5. We have that

(1) $\mathbb{Z} \mathbb{P}\left[x_{2}, x_{2}^{\prime}, \ldots, x_{n}, x_{n}^{\prime}\right]=\operatorname{Ker}(f) \oplus \mathbb{Z} \mathbb{P}^{\mathrm{st}}\left[x_{2}, x_{2}^{\prime}, \ldots, x_{n}, x_{n}^{\prime}\right]$;

(2) if $y \in \mathbb{Z} \mathbb{P}\left[x_{1}^{ \pm 1}, x_{2}, x_{2}^{\prime}, \ldots, x_{n}, x_{n}^{\prime}\right]$ and $y=\sum_{k=a}^{b} c_{k} x_{1}^{k}$ such that we have $c_{a} \neq 0$ and $c_{k} \in$ $\mathbb{Z} \mathbb{P}^{\mathrm{st}}\left[x_{2}, x_{2}^{\prime}, \ldots, x_{n}, x_{n}^{\prime}\right]$, then $\operatorname{LT}(y)=f\left(c_{a}\right) x_{1}^{a}$;

(3) $\mathbb{Z} \mathbb{P}\left[x_{1}^{ \pm 1}, x_{2}, x_{2}^{\prime}, \ldots, x_{n}, x_{n}^{\prime}\right] \cap \mathbb{Z} \mathbb{P}\left[x_{1}, x_{2}^{ \pm 1}, \ldots, x_{n}^{ \pm 1}\right]=\mathbb{Z} \mathbb{P}\left[x_{1}, x_{2}, x_{2}^{\prime}, \ldots, x_{n}, x_{n}^{\prime}\right]$.

Lemma 3.6. We have that

$$
\operatorname{Im}(f)=\mathbb{Z} \mathbb{P}\left[x_{2}, \widehat{x}_{2}, \ldots, x_{n}, \widehat{x}_{n}\right],
$$

where $\widehat{x}_{i}:=x_{i}^{\prime}$ if $b_{1 i}=0$ and $\widehat{x}_{i}:=x_{i}^{-1}$ otherwise.

Proof. By suitable and non-trivial modifications of the proof of [2, Lemma 6.6], we can prove the statement. By a direct calculation, for $j \in[2, n]$ one can show that

$$
f\left(x_{j}^{\prime}\right)=\left\{\begin{array}{llll}
x_{j}^{\prime} & \text { if } & b_{1 j}=0 \\
x_{j}^{-1} & \prod_{i=j+1}^{n} x_{i}^{b_{i j}} \prod_{i=n+1}^{m} x_{i}^{\left[b_{i j}\right]_{+}} & \text {if } & b_{1 j} \neq 0 .
\end{array}\right.
$$

The inclusion $\operatorname{Im}(f) \subseteq \mathbb{Z} \mathbb{P}\left[x_{2}, \widehat{x}_{2}, \ldots, x_{n}, \widehat{x}_{n}\right]$ is immediate. For each $j \in[2, n]$, we set $M_{j}=x_{j}^{-1} \prod_{i=j+1}^{n} x_{i}^{b_{i j}}$. Let $J=\left\{j \in[2, n] \mid b_{1 j}=0\right\}$. In order to prove the converse inclusion, it suffices to show that $x_{j}^{-1} \in \operatorname{Im}(f)$ for each $j \in[2, n]-J$. For $l_{j} \in \mathbb{Z}$, we have $\prod_{j=2}^{n}\left(M_{j}\right)^{l_{j}}=$ $\prod_{j=2}^{n}\left(x_{j}^{-1} \prod_{i=j+1}^{n} x_{i}^{b_{i j}}\right)^{l_{j}}=\prod_{j=2}^{n} x_{j}^{z_{j}}$ with $z_{j}:=-l_{j}+\sum_{t=2}^{j-1} b_{j t} l_{t}$. We define the multiplicative monoid

$$
\mathfrak{M}:=\left\{\prod_{k=2}^{n} M_{k}^{l_{k}}=\prod_{j=2}^{n} x_{j}^{z_{j}} \mid l_{k} \geq 0 \text { for } k \in[2, n], z_{j} \geq 0 \text { for } j \in J\right\} .
$$


Then we have that $\prod_{k=2}^{n}\left(M_{k}\right)^{l_{k}} \in \mathfrak{M}$ if and only if

$$
l_{k} \geq 0 \quad \text { for } \quad k \in[2, n] \quad \text { and } \quad l_{j} \leq \sum_{t=2}^{j-1} b_{j t} l_{t} \quad \text { for } \quad j \in J .
$$

For any $j \in[2, n]-J$, we obtain that $x_{j}^{-1} \in \mathfrak{M}$ by (3.2). Hence it is sufficient to prove that $\mathfrak{M} \subseteq \operatorname{Im}(f)$. Let $M:=\prod_{k=2}^{n} M_{k}^{l_{k}} \in \mathfrak{M}$. We will prove $M \in \operatorname{Im}(f)$ by induction on $\operatorname{deg}(M):=\sum_{k=2}^{n} l_{k}$. When $\operatorname{deg}(M)=0$, we have $M=1 \in \operatorname{Im}(f)$. Suppose that $\operatorname{deg}(M)>0$. Now we assume that each $M^{\prime} \in \operatorname{Im}(f)$ if $M^{\prime} \in \mathfrak{M}$ whose degree is no more than $\operatorname{deg}(M)-1$.

Let $j \in[2, n]$ be the largest integer such that $l_{j}>0$. Since $l_{j}-1<\sum_{t=2}^{j-1} b_{j t} l_{t}$ if $l_{j} \leq \sum_{t=2}^{j-1} b_{j t} l_{t}$ and $l_{j}-1 \geq 0$, we have that $M / M_{j} \in \mathfrak{M}$. By the induction hypothesis, we obtain that $M / M_{j} \in \operatorname{Im}(f)$. If $j \notin J$, then $f\left(x_{j}^{\prime}\right)=M_{j} \prod_{i=n+1}^{m} x_{i}^{\left[b_{i j}\right]_{+}}$, which implies $M_{j} \in \operatorname{Im}(f)$. It follows that $M=\left(M / M_{j}\right) M_{j} \in \operatorname{Im}(f)$. Now assume that $j \in J$. Using the fact that $b_{l k} \geq 0$ for $1 \leq k<l \leq n$, it follows that

$$
\begin{aligned}
x_{j}^{\prime} & =x_{j}^{-1} \sum_{r=0}^{d_{j}} \rho_{j, r} \prod_{i=2}^{j-1} x_{i}^{\left(r-d_{j}\right) \beta_{i j}} \prod_{i=j+1}^{n} x_{i}^{r \beta_{i j}} \prod_{i=n+1}^{m} x_{i}^{r\left[\beta_{i j}\right]_{+}+\left(d_{j}-r\right)\left[-\beta_{i j}\right]_{+}} \\
& =M_{j} \prod_{i=n+1}^{m} x_{i}^{\left[b_{i j}\right]_{+}}+x_{j}^{-1} \sum_{r=0}^{d_{j}-1} \rho_{j, r} \prod_{i=2}^{j-1} x_{i}^{\left(r-d_{j}\right) \beta_{i j}} \prod_{i=j+1}^{n} x_{i}^{r \beta_{i j}} \prod_{i=n+1}^{m} x_{i}^{r\left[\beta_{i j}\right]_{+}+\left(d_{j}-r\right)\left[-\beta_{i j}\right]_{+}} .
\end{aligned}
$$

By multiplying both sides of this equation by $M / M_{j}$, we have that

$$
\left(M / M_{j}\right) x_{j}^{\prime}=M \prod_{i=n+1}^{m} x_{i}^{\left[b_{i j}\right]_{+}}+\sum_{r=0}^{d_{j}-1} \rho_{j, r} M \prod_{i=2}^{n} x_{i}^{\left(r-d_{j}\right) \beta_{i j}} \prod_{i=n+1}^{m} x_{i}^{r\left[\beta_{i j}\right]_{+}+\left(d_{j}-r\right)\left[-\beta_{i j}\right]_{+}} .
$$

Note that $\left(M / M_{j}\right) x_{j}^{\prime} \in \operatorname{Im}(f)$. If $M \prod_{i=2}^{n} x_{i}^{\left(r-d_{j}\right) \beta_{i j}} \in \operatorname{Im}(f)$ for all $r \in\left[0, d_{j}-1\right]$, then $M \in \operatorname{Im}(f)$. Thus we only need to prove that $M \prod_{i=2}^{n} x_{i}^{\left(r-d_{j}\right) \beta_{i j}} \in \operatorname{Im}(f)$ for $r \in\left[0, d_{j}-1\right]$. Since $\prod_{i=j+1}^{n} x_{i}^{r \beta_{i j}} \in$ $\operatorname{Im}(f)$, it is sufficient to prove that

$$
M \prod_{i=j+1}^{n} x_{i}^{-b_{i j}} \prod_{i=2}^{j-1} x_{i}^{\left(r-d_{j}\right) \beta_{i j}}=\left(M / M_{j}\right)\left(x_{j}^{-1} \prod_{i=2}^{j-1} x_{i}^{\left(r-d_{j}\right) \beta_{i j}}\right) \in \operatorname{Im}(f) .
$$

For simplicity, we denote $M_{j}^{-}:=x_{j}^{-1} \prod_{i=2}^{j-1} x_{i}^{-b_{i j}}$. Then

$$
\left(M / M_{j}\right)\left(x_{j}^{-1} \prod_{i=2}^{j-1} x_{i}^{\left(r-d_{j}\right) \beta_{i j}}\right)=\left(M / M_{j}\right) M_{j}^{-} \prod_{i=2}^{j-1} x_{i}^{r \beta_{i j}}
$$

By applying the facts that $l_{j}>0$ for any $j \in J$ and (3.2), we know that there exists the smallest integer $h \in[2, j-1]$ such that $b_{j h} l_{h}>0$ which implies $b_{j h}>0, l_{h}>0$ and $b_{j k} l_{k}=0$ 
for $k \in[2, h-1]$. By $(3.2)$, we know that $h \in[2, n]-J$. Let $M^{\prime}:=\prod_{k=2}^{n} M_{k}^{l_{k}^{\prime}}$ be a monomial such that

$$
l_{k}^{\prime}= \begin{cases}0 & \text { for } \quad 2 \leq k \leq h-1, \\ 1 & \text { for } \quad k=h, \\ \min \left\{l_{k}, \sum_{t=2}^{k-1} b_{k t} l_{t}^{\prime}\right\} & \text { for } \quad h+1 \leq k \leq n .\end{cases}
$$

Note that $l_{k}^{\prime} \geq 0$ since $b_{k t} \geq 0$ for $2 \leq t<k \leq n$. By $h \in[2, n]-J$ and (3.3), we know that $l_{k}^{\prime} \leq \sum_{t=2}^{k-1} b_{k t} l_{t}^{\prime}$ for $k \in J$. Hence $M^{\prime} \in \mathfrak{M}$. It is clear that $\operatorname{deg}\left(M^{\prime}\right) \geq 1$.

In order to prove $M / M^{\prime} \in \mathfrak{M}$, we need to verify that

$$
l_{k}^{\prime} \leq l_{k} \quad \text { for } \quad k \in[2, n]
$$

and

$$
-l_{k}^{\prime}+\sum_{t=2}^{k-1} b_{k t} l_{t}^{\prime} \leq-l_{k}+\sum_{t=2}^{k-1} b_{k t} l_{t} \quad \text { for } \quad k \in J .
$$

By (3.3) and the choice of $h$, we have that the inequalities (3.4) hold.

We show that the inequalities (3.5) hold as follows:

(a) it is clear that $-l_{k}^{\prime}+\sum_{t=2}^{k-1} b_{k t} l_{t}^{\prime}=0 \leq-l_{k}+\sum_{t=2}^{k-1} b_{k t} l_{t}$ for $k \in J \cap[2, h-1]$;

(b) when $k=h$, recall that $h \notin J$;

(c) for $k \in[h+1, n]$, we have $l_{k}^{\prime}=l_{k}$ or $l_{k}^{\prime}=\sum_{t=2}^{k-1} b_{k t} l_{t}^{\prime}$. If $l_{k}^{\prime}=l_{k}$, then the inequalities (3.5) hold since $l_{t}^{\prime} \leq l_{t}$ for $2 \leq t \leq k-1$. If $l_{k}^{\prime}=\sum_{t=2}^{k-1} b_{k t} l_{t}^{\prime}$, then $-l_{k}^{\prime}+\sum_{t=2}^{k-1} b_{k t} l_{t}^{\prime}=0 \leq-l_{k}+\sum_{t=2}^{k-1} b_{k t} l_{t}$ for $k \in J \cap[h+1, n]$. Thus we obtain the inequalities (3.5).

The inequalities (3.4) and (3.5) imply that $M / M^{\prime} \in \mathfrak{M}$. By (3.4), we have that $\operatorname{deg}\left(M / M^{\prime}\right)<$ $\operatorname{deg}(M)$. The induction hypothesis implies that $M / M^{\prime} \in \operatorname{Im}(f)$.

Note that $\left(M / M_{j}\right) M_{j}^{-} \prod_{k=2}^{j-1} x_{k}^{r \beta_{k j}}=\left(M / M^{\prime}\right)\left(M^{\prime} M_{j}^{-} / M_{j}\right) \prod_{k=2}^{j-1} x_{k}^{r \beta_{k j}}$. Since we know that $M / M^{\prime} \in \operatorname{Im}(f)$, it suffices to show that $\left(M^{\prime} M_{j}^{-} / M_{j}\right) \prod_{k=2}^{j-1} x_{k}^{r \beta_{k j}} \in \operatorname{Im}(f)$.

Let $\prod_{k=2}^{n} x_{k}^{c_{k}}=\left(M^{\prime} M_{j}^{-} / M_{j}\right) \prod_{t=2}^{j-1} x_{t}^{r \beta_{t j}}$ with $c_{k} \in \mathbb{Z}$ for all $k \in[2, n]$. To prove that $\prod_{k=2}^{n} x_{k}^{c_{k}} \in$ $\operatorname{Im}(f)$, we only need to show that $c_{k} \geq 0$ for all $k \in[2, n]$. Note that $0 \leq r \leq d_{j}-1$.

By calculating the powers of $x_{k}$ in $M^{\prime}, M_{j}^{-}, M_{j}$ and $\prod_{t=2}^{j-1} x_{t}^{r \beta_{t j}}$, respectively, it follows that:

(1) when $k \in[2, h-1]$, we have $\beta_{k j} \leq 0$ and

$$
c_{k}=0+\left(-b_{k j}\right)-0+r \beta_{k j}=\left(r-d_{j}\right) \beta_{k j} \geq 0
$$

(2) when $k=h$, since $b_{j h}>0$, we obtain $\beta_{h j}<0$. It follows that

$$
c_{h}=-l_{h}^{\prime}+\left(-b_{h j}\right)-0+r \beta_{h j}=-1+\left(r-d_{j}\right) \beta_{h j} \geq-\beta_{h j}-1 \geq 0
$$


(3) when $k \in[h+1, j-1]$, then $\beta_{k j} \leq 0$ and by (3.3) we have that

$$
c_{k}=\left(-l_{k}^{\prime}+\sum_{t=2}^{k-1} b_{k t} l_{t}^{\prime}\right)+0-b_{k j}+r \beta_{k j} \geq\left(r-d_{j}\right) \beta_{k j} \geq 0 ;
$$

(4) when $k=j$, by (3.3) we obtain that

$$
c_{j}=\left(-l_{j}^{\prime}+\sum_{t=2}^{j-1} b_{j t} l_{t}^{\prime}\right)+(-1)-(-1)+0=-l_{j}^{\prime}+\sum_{t=2}^{j-1} b_{j t} l_{t}^{\prime} \geq 0
$$

(5) when $k \in[j+1, n]$, note that $l_{k}=0$. But $0 \leq l_{k}^{\prime} \leq l_{k}$ which implies $l_{k}^{\prime}=0$. Since $\sum_{t=2}^{j-1} b_{j t} l_{t}^{\prime} \geq b_{j h} l_{h}^{\prime}=b_{j h}>0$ and $l_{j}>0$, we have $l_{j}^{\prime}>0$. It follows that

$$
c_{k}=\left(-l_{k}^{\prime}+\sum_{t=2}^{k-1} b_{k t} l_{t}^{\prime}\right)+0-b_{k j}+0=\sum_{t=2}^{k-1} b_{k t} l_{t}^{\prime}-b_{k j} \geq b_{k j}\left(l_{j}^{\prime}-1\right) \geq 0 .
$$

Hence $c_{k} \geq 0$ for all $k \in[2, n]$. The proof is completed.

Remark 3.7. In the ordinary cluster algebras, the exchange relations are binomial relations, but the exchange relations of the generalized cluster algebras are polynomial relations. Therefore we need more detailed discussions. For example, in the proof of Lemma 3.6 we should choose the smallest integer $h \in[2, j-1]$ such that $b_{j h} l_{h}>0$ which implies $b_{j h}>0, l_{h}>0$ and $b_{j k} l_{k}=0$ for $k \in[2, h-1]$. The choice of such $h$ makes the most significant difference between our proof and the proof in [2].

In order to prove Theorem 3.10 below, we need the following two lemmas.

Lemma 3.8. Let $(\widetilde{\mathbf{x}}, \rho, \widetilde{B})$ be acyclic and coprime. For $j \in J=\left\{j \in[2, n] \mid b_{1 j}=0\right\}$, if $z \in$ $\mathbb{Z P}\left[x_{2}^{ \pm 1}, \ldots, x_{n}^{ \pm 1}\right]$ and $z P_{1} \in \mathbb{Z} \mathbb{P}\left[x_{2}^{ \pm 1}, \ldots, x_{j-1}^{ \pm 1}, x_{j}, x_{j}^{\prime}, x_{j+1}^{ \pm 1}, \ldots, x_{n}^{ \pm 1}\right]$, then

$$
z \in \mathbb{Z} \mathbb{P}\left[x_{2}^{ \pm 1}, \ldots, x_{j-1}^{ \pm 1}, x_{j}, x_{j}^{\prime}, x_{j+1}^{ \pm 1}, \ldots, x_{n}^{ \pm 1}\right] .
$$

Proof. For each $j \in J$, we can write the element $z$ in the form $z=\sum_{k=a_{j}}^{b_{j}} c_{j, k} x_{j}^{k}$ with $c_{j, k} \in$ $\mathbb{Z} \mathbb{P}\left[x_{2}^{ \pm 1}, \ldots, x_{j-1}^{ \pm 1}, x_{j+1}^{ \pm 1}, \ldots, x_{n}^{ \pm 1}\right]$ and $c_{j, a_{j}} \neq 0$. If $a_{j} \geq 0$, then we have that

$$
z \in \mathbb{Z} \mathbb{P}\left[x_{2}^{ \pm 1}, \ldots, x_{j-1}^{ \pm 1}, x_{j}, x_{j+1}^{ \pm 1}, \ldots, x_{n}^{ \pm 1}\right] .
$$

Now assume that $a_{j}<0$. The fact $z P_{1} \in \operatorname{Im}(f)$ implies that

$$
c_{k} x_{j}^{k} P_{1}=c_{k} P_{j}^{-|k|}\left(x_{j}^{\prime}\right)^{|k|} P_{1} \in \mathbb{Z} \mathbb{P}\left[x_{2}^{ \pm 1}, \ldots, x_{j-1}^{ \pm 1}, x_{j}, x_{j}^{\prime}, x_{j+1}^{ \pm 1}, \ldots, x_{n}^{ \pm 1}\right]
$$

for $k<0$. Since $P_{1}$ and $P_{j}$ are coprime, we have that

$$
c_{k} P_{j}^{-|k|} \in \mathbb{Z} \mathbb{P}\left[x_{2}^{ \pm 1}, \ldots, x_{j-1}^{ \pm 1}, x_{j}, x_{j}^{\prime}, x_{j+1}^{ \pm 1}, \ldots, x_{n}^{ \pm 1}\right] .
$$

The proof is completed.

The following result shows that the upper bound of the generalized cluster algebra of rank 2 is equal to the corresponding lower bound. 
Lemma 3.9. Suppose that $n=2$. If the generalized seed $(\widetilde{\mathbf{x}}, \rho, \widetilde{B})$ is coprime, then

$$
\mathbb{Z} \mathbb{P}\left[x_{1}^{ \pm 1}, x_{2}, x_{2}^{\prime}\right] \cap \mathbb{Z} \mathbb{P}\left[x_{1}, x_{1}^{\prime}, x_{2}^{ \pm 1}\right]=\mathbb{Z} \mathbb{P}\left[x_{1}, x_{1}^{\prime}, x_{2}, x_{2}^{\prime}\right] .
$$

Proof. Obviously, we have that $\mathbb{Z P}\left[x_{1}^{ \pm 1}, x_{2}, x_{2}^{\prime}\right] \cap \mathbb{Z} \mathbb{P}\left[x_{1}, x_{1}^{\prime}, x_{2}^{ \pm 1}\right] \supseteq \mathbb{Z} \mathbb{P}\left[x_{1}, x_{1}^{\prime}, x_{2}, x_{2}^{\prime}\right]$. Let $y \in$ $\mathbb{Z} \mathbb{P}\left[x_{1}^{ \pm 1}, x_{2}, x_{2}^{\prime}\right] \cap \mathbb{Z} \mathbb{P}\left[x_{1}, x_{1}^{\prime}, x_{2}^{ \pm 1}\right]$. Note that the element $y$ can be written as $y=\sum_{k=a}^{b} c_{k} x_{1}^{k}$ with $c_{k} \in \mathbb{Z} \mathbb{P}^{\text {st }}\left[x_{2}, x_{2}^{\prime}\right]$ and $c_{a} \neq 0$. By Lemma $3.5(3)$, if the leading term $\operatorname{LT}(y)=f\left(c_{a}\right) x_{1}^{a} \in$ $\mathbb{Z} \mathbb{P}\left[x_{1}, x_{2}^{ \pm 1}\right]$, i.e., $a>0$, then $y \in \mathbb{Z} \mathbb{P}\left[x_{1}, x_{2}, x_{2}^{\prime}\right]$.

Suppose that $a \leq 0$. We will prove (3.6) by induction on $-a$. It is enough to find an element $y^{\prime} \in \mathbb{Z} \mathbb{P}\left[x_{1}, x_{1}^{\prime}, x_{2}, x_{2}^{\prime}\right]$ such that $\operatorname{LT}(y)=\operatorname{LT}\left(y^{\prime}\right)$. By Lemma 3.4 , we have $f\left(c_{a}\right) P_{1}^{a} \in \mathbb{Z} \mathbb{P}\left[x_{2}^{ \pm 1}\right]$, i.e., there exists some element $z \in \mathbb{Z} \mathbb{P}\left[x_{2}^{ \pm 1}\right]$ such that $f\left(c_{a}\right)=z P_{1}^{-a}$. By Lemma 3.6, if $b_{12}=0$ then $z \in \mathbb{Z} \mathbb{P}\left[x_{2}^{ \pm 1}\right]=\operatorname{Im}(f)$ and if $b_{12} \neq 0$ then $\operatorname{Im}(f)=\mathbb{Z} \mathbb{P}\left[x_{2}, x_{2}^{\prime}\right]$. Note that $P_{1}$ and $P_{2}$ are coprime. By applying Lemma 3.8 repeatedly, we conclude that $z \in \mathbb{Z} \mathbb{P}\left[x_{2}, x_{2}^{\prime}\right]=\operatorname{Im}(f)$. It follows that there exists some element $z^{\prime} \in \mathbb{Z} \mathbb{P}\left[x_{2}, x_{2}^{\prime}\right]$ such that $z=f\left(z^{\prime}\right)$. Let $y^{\prime}=z^{\prime}\left(x_{1}^{\prime}\right)^{-a}$, then we have that $y^{\prime} \in \mathbb{Z} \mathbb{P}\left[x_{1}, x_{1}^{\prime}, x_{2}, x_{2}^{\prime}\right]$ and $\operatorname{LT}\left(y^{\prime}\right)=f\left(z^{\prime}\right) P_{1}^{-a} x_{1}^{a}=z P_{1}^{-a} x_{1}^{a}=f\left(c_{a}\right) x_{1}^{a}=\operatorname{LT}(y)$. Hence the absolute value of the power of $x_{1}$ in $y-y^{\prime}$ is strictly less than $-a$. We see that $y-y^{\prime} \in \mathbb{Z} \mathbb{P}\left[x_{1}, x_{1}^{\prime}, x_{2}, x_{2}^{\prime}\right]$ by the induction hypothesis. Therefore $y \in \mathbb{Z} \mathbb{P}\left[x_{1}, x_{1}^{\prime}, x_{2}, x_{2}^{\prime}\right]$, as desired. The proof is completed.

Theorem 3.10. If the generalized seed $(\widetilde{\mathbf{x}}, \rho, \widetilde{B})$ is coprime and acyclic, then

$$
\mathcal{L}(\widetilde{\mathbf{x}}, \rho, \widetilde{B})=\mathcal{U}(\widetilde{\mathbf{x}}, \rho, \widetilde{B}) .
$$

Proof. Let $n$ be the rank of $\mathcal{A}(\widetilde{\mathbf{x}}, \rho, \widetilde{B})$. We prove the statement by induction on $n$. We denote $\mathcal{U}:=\mathcal{U}(\widetilde{\mathbf{x}}, \rho, \widetilde{B})$ and $\mathcal{L}:=\mathcal{L}(\widetilde{\mathbf{x}}, \rho, \widetilde{B})$ for simplification purposes. When $n=1$, by using (3.1), we have $\mathcal{L}=\mathcal{U}$. When $n=2$, the statement follows by Lemmas 3.3 and 3.9. Now let $n \geq 3$ and assume that the upper bound coincides with the lower bound if the rank of the acyclic and coprime generalized cluster algebra is less than $n$. By Lemma 3.3, we have that

$$
\mathcal{U}=\mathbb{Z} \mathbb{P}\left[x_{1}, x_{1}^{\prime}, x_{2}^{ \pm 1}, \ldots, x_{n}^{ \pm 1}\right] \cap \bigcap_{i=2}^{n} \mathbb{Z} \mathbb{P}\left[x_{1}^{ \pm 1}, \ldots, x_{i-1}^{ \pm 1}, x_{i}, x_{i}^{\prime}, x_{i+1}^{ \pm 1}, \ldots, x_{n}^{ \pm 1}\right] .
$$

Let the generalized seed $\left(\widetilde{\mathbf{x}}^{\dagger}, \rho^{\dagger}, \widetilde{B}^{\dagger}\right)$ be the freezing of $(\widetilde{\mathbf{x}}, \rho, \widetilde{B})$ at $x_{1}$. It follows that $\mathbb{Z} \mathbb{P}^{\dagger}=$ $\mathbb{Z} \mathbb{P}\left[x_{1}^{ \pm 1}\right]$. For $i \in[2, n]$, we have that

$$
\mathbb{Z} \mathbb{P}^{\dagger}\left[x_{2}^{ \pm 1}, \ldots, x_{i-1}^{ \pm 1}, x_{i}, x_{i}^{\prime}, x_{i+1}^{ \pm 1}, \ldots, x_{n}^{ \pm 1}\right]=\mathbb{Z} \mathbb{P}\left[x_{1}^{ \pm 1}, \ldots, x_{i-1}^{ \pm 1}, x_{i}, x_{i}^{\prime}, x_{i+1}^{ \pm 1}, \ldots, x_{n}^{ \pm 1}\right] .
$$

By the induction hypothesis, we obtain that

$$
\bigcap_{i=2}^{n} \mathbb{Z} \mathbb{P}^{\dagger}\left[x_{2}^{ \pm 1} \ldots, x_{i-1}^{ \pm 1}, x_{i}, x_{i}^{\prime}, x_{i+1}^{ \pm 1}, \ldots, x_{n}^{ \pm 1}\right]=\mathbb{Z P}^{\dagger}\left[x_{2}, x_{2}^{\prime}, \ldots, x_{n}, x_{n}^{\prime}\right] .
$$

Thus it is enough to prove that

$$
\mathbb{Z} \mathbb{P}\left[x_{1}^{ \pm 1}, x_{2}, x_{2}^{\prime}, \ldots, x_{n}, x_{n}^{\prime}\right] \cap \mathbb{Z} \mathbb{P}\left[x_{1}, x_{1}^{\prime}, x_{2}^{ \pm 1}, \ldots, x_{n}^{ \pm 1}\right]=\mathbb{Z} \mathbb{P}\left[x_{1}, x_{1}^{\prime}, \ldots, x_{n}, x_{n}^{\prime}\right] .
$$

The proof of (3.7) is quite similar to that given earlier for (3.6). It is easy to see that the " $\supseteq$ " part holds. We only need to show the "ᄃ" part. Assume

$$
y \in \mathbb{Z} \mathbb{P}\left[x_{1}^{ \pm 1}, x_{2}, x_{2}^{\prime}, \ldots, x_{n}, x_{n}^{\prime}\right] \cap \mathbb{Z} \mathbb{P}\left[x_{1}, x_{1}^{\prime}, x_{2}^{ \pm 1}, \ldots, x_{n}^{ \pm 1}\right] .
$$

We can write $y=\sum_{k=a}^{b} c_{k} x_{1}^{k}$ with $c_{k} \in \mathbb{Z P}^{\mathrm{st}}\left[x_{2}, x_{2}^{\prime}, \ldots, x_{n}, x_{n}^{\prime}\right]$ and $c_{a} \neq 0$. 
If $\operatorname{LT}(y) \in \mathbb{Z} \mathbb{P}\left[x_{1}, x_{2}^{ \pm 1}, \ldots, x_{n}^{ \pm 1}\right]$, we have $y \in \mathbb{Z} \mathbb{P}\left[x_{1}, x_{2}, x_{2}^{\prime}, \ldots, x_{n}, x_{n}^{\prime}\right]$ by Lemma $3.5(3)$ and the statement is true. Hence we can assume that $a<0$. We prove (3.7) by induction on $|a|$. It suffices to prove that there exists some element $y_{1} \in \mathbb{Z} \mathbb{P}\left[x_{1}, x_{1}^{\prime}, x_{2}, x_{2}^{\prime}, \ldots, x_{n}, x_{n}^{\prime}\right]$ such that $\operatorname{LT}(y)=\operatorname{LT}\left(y_{1}\right)$. Set $z=f\left(c_{a}\right) P_{1}^{a}$. By Lemma 3.4, we have that $z=f\left(c_{a}\right) P_{1}^{a} \in$ $\mathbb{Z} \mathbb{P}\left[x_{2}^{ \pm 1}, \ldots, x_{n}^{ \pm 1}\right]$. Note that we have $z P_{1}^{-a} \in \operatorname{Im}(f)$. We claim that $z \in \operatorname{Im}(f)$. By Lemma 3.6, if $J=\varnothing$, then the claim is true. Suppose that $J \neq \varnothing$. By applying Lemma 3.8 repeatedly, we conclude that $z \in \bigcap_{j \in J} \mathbb{Z} \mathbb{P}\left[x_{2}^{ \pm 1}, \ldots, x_{j-1}^{ \pm 1}, x_{j}, x_{j}^{\prime}, x_{j+1}^{ \pm 1}, \ldots, x_{n}^{ \pm 1}\right]$. It remains to show that

$$
\bigcap_{j \in J} \mathbb{Z} \mathbb{P}\left[x_{2}^{ \pm 1}, \ldots, x_{j-1}^{ \pm 1}, x_{j}, x_{j}^{\prime}, x_{j+1}^{ \pm 1}, \ldots, x_{n}^{ \pm 1}\right]=\operatorname{Im}(f) .
$$

We obtain the generalized seed $(\widetilde{\mathbf{y}}, \varepsilon, \widetilde{A})$ from $(\widetilde{\mathbf{x}}, \rho, \widetilde{B})$ through freezing at $\left\{x_{j} \mid j \in[2, n]-J\right\}$ and then removing the cluster variable $x_{1}$. The group ring of coefficients of $\mathcal{A}(\widetilde{\mathbf{y}}, \varepsilon, \widetilde{A})$ is $\mathbb{Z} \mathbb{P}\left[x_{j}^{ \pm 1} \mid j \in\right.$ $[2, n]-J]$. By the induction hypothesis, we have that $\mathcal{L}(\widetilde{\mathbf{y}}, \varepsilon, \widetilde{A})=\mathcal{U}(\widetilde{\mathbf{y}}, \varepsilon, \widetilde{A})$, namely

$$
\bigcap_{j \in J} \mathbb{Z} \mathbb{P}\left[x_{2}^{ \pm 1}, \ldots, x_{j-1}^{ \pm 1}, x_{j}, x_{j}^{\prime}, x_{j+1}^{ \pm 1}, \ldots, x_{n}^{ \pm 1}\right]=\mathbb{Z} \mathbb{P}\left[x_{2}, \widehat{x}_{2}, \ldots, x_{n}, \widehat{x}_{n}\right]=\operatorname{Im}(f) .
$$

Thus $z \in \operatorname{Im}(f)$ and there exists some $z_{1} \in \mathbb{Z} \mathbb{P}\left[x_{2}, x_{2}^{\prime}, \ldots, x_{n}, x_{n}^{\prime}\right]$ such that $z=f\left(z_{1}\right)$. Let $y_{1}=$ $z_{1}\left(x_{1}^{\prime}\right)^{|a|}$. Then $\operatorname{LT}\left(y_{1}\right)=\operatorname{LT}(y)$. This completes the proof.

The following result follows immediately from Theorems 3.1 and 3.10.

Corollary 3.11. If the generalized seed $(\widetilde{\mathbf{x}}, \rho, \widetilde{B})$ is acyclic and coprime, then the standard monomials in $\left\{x_{1}, x_{1}^{\prime}, \ldots, x_{n}, x_{n}^{\prime}\right\}$ form a $\mathbb{Z} \mathbb{P}$-basis of $\mathcal{A}(\widetilde{\mathbf{x}}, \rho, \widetilde{B})$.

For convenience, the basis consisting of the standard monomials is called the standard monomial basis.

\section{A Generalized upper cluster algebras}

In this appendix, we prove that every acyclic generalized cluster algebra coincides with its corresponding upper cluster algebra without the assumption of the existence of coprimality. We mimic the proof of the main results given in [12].

Definition A.1. Let $(\widetilde{\mathbf{x}}, \rho, \widetilde{B})$ be a generalized seed. The generalized upper cluster algebra of $\mathcal{A}(\widetilde{\mathbf{x}}, \rho, \widetilde{B})$ is defined as

$$
\widetilde{\mathcal{U}}(\widetilde{\mathbf{x}}, \rho, \widetilde{B}):=\bigcap_{(\widetilde{\mathbf{y}}, \varepsilon, \widetilde{A}) \sim(\widetilde{\mathbf{x}}, \rho, \widetilde{B})} \mathbb{Z P}\left[y_{1}^{ \pm 1}, \ldots, y_{n}^{ \pm 1}\right],
$$

where the generalized seed $(\widetilde{\mathbf{y}}, \varepsilon, \widetilde{A})$ is mutation-equivalent to $(\widetilde{\mathbf{x}}, \rho, \widetilde{B})$.

Obviously, the generalized upper cluster algebra $\widetilde{\mathcal{U}}(\widetilde{\mathbf{x}}, \rho, \widetilde{B})$ is contained in the corresponding upper bound.

Lemma A.2. Let $\left\{x_{i_{1}}, \ldots, x_{i_{k}}\right\} \subsetneq \mathbf{x}$. If $\left(\widetilde{\mathbf{x}}^{\dagger}, \rho^{\dagger}, \widetilde{B}^{\dagger}\right)$ is the freezing of $(\widetilde{\mathbf{x}}, \rho, \widetilde{B})$ at the set $\left\{x_{i_{1}}, \ldots, x_{i_{k}}\right\}$, then we have that

$$
\mathcal{A}\left(\widetilde{\mathbf{x}}^{\dagger}, \rho^{\dagger}, \widetilde{B}^{\dagger}\right) \subseteq \mathcal{A}(\widetilde{\mathbf{x}}, \rho, \widetilde{B})\left[\left(x_{i_{1}} \cdots x_{i_{k}}\right)^{-1}\right] \subseteq \widetilde{\mathcal{U}}(\widetilde{\mathbf{x}}, \rho, \widetilde{B})\left[\left(x_{i_{1}} \cdots x_{i_{k}}\right)^{-1}\right] \subseteq \widetilde{\mathcal{U}}\left(\widetilde{\mathbf{x}}^{\dagger}, \rho^{\dagger}, \widetilde{B}^{\dagger}\right) .
$$

Proof. By the definitions of the freezing of generalized cluster algebras and the generalized upper cluster algebras, the first and the third inclusions are immediate. By the Laurent phenomenon, i.e., $\mathcal{A}(\widetilde{\mathbf{x}}, \rho, \widetilde{B}) \subseteq \widetilde{\mathcal{U}}(\widetilde{\mathbf{x}}, \rho, \widetilde{B})$, we obtain the second inclusion. 
Definition A.3. Let $\left(\widetilde{\mathbf{x}}^{\dagger}, \rho^{\dagger}, \widetilde{B}^{\dagger}\right)$ be the freezing of $(\widetilde{\mathbf{x}}, \rho, \widetilde{B})$ at $\left\{x_{i_{1}}, \ldots, x_{i_{k}}\right\}$. If

$$
\mathcal{A}\left(\widetilde{\mathbf{x}}^{\dagger}, \rho^{\dagger}, \widetilde{B}^{\dagger}\right)=\mathcal{A}(\widetilde{\mathbf{x}}, \rho, \widetilde{B})\left[\left(x_{i_{1}} \cdots x_{i_{k}}\right)^{-1}\right]
$$

then we call $\mathcal{A}\left(\widetilde{\mathbf{x}}^{\dagger}, \rho^{\dagger}, \widetilde{B}^{\dagger}\right)$ a cluster localization of $\mathcal{A}(\widetilde{\mathbf{x}}, \rho, \widetilde{B})$.

If $\mathcal{A}\left(\widetilde{\mathbf{x}}^{\dagger}, \rho^{\dagger}, \widetilde{B}^{\dagger}\right)$ is a cluster localization of $\mathcal{A}(\widetilde{\mathbf{x}}, \rho, \widetilde{B})$ and $\mathcal{A}\left(\widetilde{\mathbf{x}}^{\ddagger}, \rho^{\ddagger}, \widetilde{B}^{\ddagger}\right)$ a cluster localization of $\mathcal{A}\left(\widetilde{\mathbf{x}}^{\dagger}, \rho^{\dagger}, \widetilde{B}^{\dagger}\right)$, then $\mathcal{A}\left(\widetilde{\mathbf{x}}^{\ddagger}, \rho^{\ddagger}, \widetilde{B}^{\ddagger}\right)$ is also a cluster localization of $\mathcal{A}(\widetilde{\mathbf{x}}, \rho, \widetilde{B})$.

Definition A.4. Let $\mathcal{A}(\widetilde{\mathbf{x}}, \rho, \widetilde{B})$ be a generalized cluster algebra. Let $\left\{\mathcal{A}_{i} \mid i \in I\right\}$ be the set such that $\mathcal{A}_{i}$ are the cluster localizations of $\mathcal{A}(\widetilde{\mathbf{x}}, \rho, \widetilde{B})$. For each prime ideal $\mathfrak{p}$ of $\mathcal{A}(\widetilde{\mathbf{x}}, \rho, \widetilde{B})$, if there exists some $i \in I$ such that $\mathfrak{p} \mathcal{A}_{i} \subsetneq \mathcal{A}_{i}$, then the set $\left\{\mathcal{A}_{i} \mid i \in I\right\}$ is called a cover of $\mathcal{A}(\widetilde{\mathbf{x}}, \rho, \widetilde{B})$.

Example A.5. Let $(\mathbf{x}, \rho, B)$ be the generalized seed from Example 2.3. The freezing of $\mathcal{A}(\mathbf{x}, \rho, B)$ at $x_{1}$ is $\mathcal{A}_{1}:=\mathbb{Z}\left[x_{1}^{ \pm 1}, x_{2}, \frac{x_{1}+1}{x_{2}}\right]=\mathcal{A}(\mathbf{x}, \rho, B)\left[x_{1}^{-1}\right]$, and the freezing at $x_{2}$ is $\mathcal{A}_{2}:=\mathbb{Z}\left[x_{1}, x_{2}^{ \pm 1}, \frac{1+h x_{2}+x_{2}^{2}}{x_{1}}\right]=\mathcal{A}(\mathbf{x}, \rho, B)\left[x_{2}^{-1}\right]$. Since the ideal $\left(x_{1}, x_{2}\right)=\mathcal{A}(\mathbf{x}, \rho, B)$, we conclude that $\left\{x_{1}, x_{2}\right\} \nsubseteq \mathfrak{p}$ for any prime ideal $\mathfrak{p}$ of $\mathcal{A}(\mathbf{x}, \rho, B)$. It follows that $\left\{\mathcal{A}_{1}, \mathcal{A}_{2}\right\}$ is a cover of $\mathcal{A}(\mathbf{x}, \rho, B)$.

Lemma A.6. If $\mathcal{A}(\widetilde{\mathbf{x}}, \rho, \widetilde{B})$ has a cover $\left\{\mathcal{A}_{i} \mid i \in I\right\}$ and each $\mathcal{A}_{i}$ has a cover $\left\{\mathcal{A}_{i j} \mid j \in J_{i}\right\}$, then $\left\{\mathcal{A}_{i j} \mid i \in I, j \in J_{i}\right\}$ is a cover of $\mathcal{A}(\widetilde{\mathbf{x}}, \rho, \widetilde{B})$. Namely, the covers are transitive.

Proof. Let $\mathfrak{p}$ be a prime ideal of $\mathcal{A}(\widetilde{\mathbf{x}}, \rho, \widetilde{B})$, and suppose there exists some $\mathcal{A}_{i}$ such that $\mathfrak{p} \mathcal{A}_{i} \subsetneq \mathcal{A}_{i}$. Since $\mathfrak{p} \mathcal{A}_{i}$ is a proper ideal of $\mathcal{A}_{i}$, there exists a maximal ideal $\mathfrak{m}$ in $\mathcal{A}_{i}$ such that $\mathfrak{p} \mathcal{A}_{i} \subseteq \mathfrak{m}$. There exists some $A_{i j}$ such that $\mathfrak{m} \mathcal{A}_{i j} \subsetneq \mathcal{A}_{i j}$. Therefore we have that $\mathfrak{p} \mathcal{A}_{i j} \subseteq$ $\mathfrak{m} \mathcal{A}_{i j} \subsetneq \mathcal{A}_{i j}$. This completes the proof.

Lemma A.7. Let $\left\{\mathcal{A}_{i} \mid i \in I\right\}$ be a cover of the generalized cluster algebra $\mathcal{A}(\widetilde{\mathbf{x}}, \rho, \widetilde{B})$. Let $\widetilde{\mathcal{U}}_{i}$ denote the generalized upper cluster algebra of $\mathcal{A}_{i}$ for each $i \in I$. We have

(1) $\mathcal{A}(\widetilde{\mathbf{x}}, \rho, \widetilde{B})=\bigcap_{i \in I} \mathcal{A}_{i}$;

(2) if $\mathcal{A}_{i}=\widetilde{\mathcal{U}}_{i}$ for all $i \in I$, then $\mathcal{A}(\widetilde{\mathbf{x}}, \rho, \widetilde{B})=\widetilde{\mathcal{U}}(\widetilde{\mathbf{x}}, \rho, \widetilde{B})$.

Proof. The proof of the lemma is quite similar to the one used in [12, Proposition 2, Lemma 2], so is omitted.

A generalized cluster algebra $\mathcal{A}(\widetilde{\mathbf{x}}, \rho, \widetilde{B})$ is called isolated if the principal part $B=0$.

Proposition A.8. If $\mathcal{A}(\widetilde{\mathbf{x}}, \rho, \widetilde{B})$ is an isolated generalized cluster algebra, then we have that $\mathcal{A}(\widetilde{\mathbf{x}}, \rho, \widetilde{B})=\widetilde{\mathcal{U}}(\widetilde{\mathbf{x}}, \rho, \widetilde{B})$.

Proof. Proving the proposition uses the same ideas in [12, Proposition 3].

Proposition A.9. If the generalized cluster algebra $\mathcal{A}(\widetilde{\mathbf{x}}, \rho, \widetilde{B})$ is acyclic, then it has a cover $\left\{\mathcal{A}_{i} \mid i \in I\right\}$ with each $\mathcal{A}_{i}$ being isolated.

Proof. We can assume that $b_{i j} \geq 0$ for $i, j \in[1, n]$ with $i>j$. There exist $k, l \in[1, n]$ such that $l$ is a source in $\Gamma(\widetilde{\mathbf{x}}, \rho, \widetilde{B})$ and $b_{k l}<0$. Recall the exchange relation

$$
x_{l} x_{l}^{\prime}=\sum_{r=0}^{d_{l}-1} \rho_{l, r} \prod_{i=1}^{m} x_{i}^{r\left[\beta_{i l}\right]_{+}+\left(d_{l}-r\right)\left[-\beta_{i l}\right]_{+}}+\prod_{i=n+1}^{m} x_{i}^{\left[b_{i l}\right]_{+}} .
$$


It follows that

$$
1=x_{l} x_{l}^{\prime} \prod_{i=n+1}^{m} x_{i}^{-\left[b_{i l}\right]_{+}}-\sum_{r=0}^{d_{l}-1} \rho_{l, r} \prod_{i=1}^{m} x_{i}^{r\left[\beta_{i l}\right]_{+}+\left(d_{l}-r\right)\left[-\beta_{i l}\right]_{+}} \prod_{i=n+1}^{m} x_{i}^{-\left[b_{i l}\right]_{+}} .
$$

Since $\beta_{k l}<0$, the variables $x_{k}$ and $x_{l}$ appear in the right hand side of the above equation. Thus the ideal $\left(x_{k}, x_{l}\right)$ is equal to $\mathcal{A}(\widetilde{\mathbf{x}}, \rho, \widetilde{B})$. The remainder of the proof follows the one in [12, Proposition 4].

Corollary A.10. If the generalized cluster algebra $\mathcal{A}(\widetilde{\mathbf{x}}, \rho, \widetilde{B})$ is acyclic, then we have that $\mathcal{A}(\widetilde{\mathbf{x}}, \rho, \widetilde{B})=\widetilde{\mathcal{U}}(\widetilde{\mathbf{x}}, \rho, \widetilde{B})$.

\section{Acknowledgements}

The authors are greatly indebted to referees for their valuable comments and recommendations which definitely help to improve the readability and quality of the paper. Liqian Bai was supported by NSF of China (No. 11801445), the Natural Science Foundation of Shaanxi Province (No. 2020JQ-116) and the Fundamental Research Funds for the Central Universities (No. 310201911cx027), Ming Ding was supported by NSF of China (No. 11771217) and Fan Xu was supported by NSF of China (No. 11471177).

\section{References}

[1] Benito A., Muller G., Rajchgot J., Smith K.E., Singularities of locally acyclic cluster algebras, Algebra Number Theory 9 (2015), 913-936, arXiv:1404.4399.

[2] Berenstein A., Fomin S., Zelevinsky A., Cluster algebras. III. Upper bounds and double Bruhat cells, Duke Math. J. 126 (2005), 1-52, arXiv:math.RT/0305434.

[3] Bucher E., Machacek J., Shapiro M., Upper cluster algebras and choice of ground ring, Sci. China Math. 62 (2019), 1257-1266, arXiv:1802.04835.

[4] Chekhov L., Shapiro M., Teichmüller spaces of Riemann surfaces with orbifold points of arbitrary order and cluster variables, Int. Math. Res. Not. 2014 (2014), 2746-2772, arXiv:1111.3963.

[5] Fomin S., Zelevinsky A., Cluster algebras. I. Foundations, J. Amer. Math. Soc. 15 (2002), 497-529, arXiv:math.RT/0104151.

[6] Fomin S., Zelevinsky A., Cluster algebras. II. Finite type classification, Invent. Math. 154 (2003), 63-121, arXiv:math.RA/0208229.

[7] Gekhtman M., Shapiro M., Vainshtein A., Drinfeld double of $\mathrm{GL}_{n}$ and generalized cluster structures, Proc. Lond. Math. Soc. 116 (2018), 429-484, arXiv:1605.05705.

[8] Gross M., Hacking P., Keel S., Birational geometry of cluster algebras, Algebr. Geom. 2 (2015), 137-175, arXiv:1309.2573.

[9] Lee K., Li L., Mills M.R., A combinatorial formula for certain elements of upper cluster algebras, SIGMA 11 (2015), 049, 24 pages, arXiv:1409.8177.

[10] Matherne J.P., Muller G., Computing upper cluster algebras, Int. Math. Res. Not. 2015 (2015), 3121-3149, arXiv:1307.0579.

[11] Muller G., Locally acyclic cluster algebras, Adv. Math. 233 (2013), 207-247, arXiv:1111.4468.

[12] Muller G., $\mathcal{A}=\mathcal{U}$ for locally acyclic cluster algebras, SIGMA 10 (2014), 094, 8 pages, arXiv:1308.1141.

[13] Nakanishi T., Structure of seeds in generalized cluster algebras, Pacific J. Math. 277 (2015), 201-217, arXiv:1409.5967.

[14] Plamondon P.-G., Generic bases for cluster algebras from the cluster category, Int. Math. Res. Not. 2013 (2013), 2368-2420, arXiv:1111.4431. 